\title{
Influence of Anthropometric Parameters and Biochemical Markers of Bone Metabolism on Quantitative Ultrasound of Bone in the Institutionalized Elderly
}

\author{
M.-A. Krieg ${ }^{1}$, J. Cornuz ${ }^{2}$, A. F. Jacquet ${ }^{1}$, D. Thiébaud ${ }^{1}$ and P. Burckhardt ${ }^{1}$ \\ ${ }^{1}$ Department of Internal Medicine, University Hospital, Lausanne; and ${ }^{2}$ Institute of Social and Preventive Medicine, Lausanne, \\ Switzerland
}

\begin{abstract}
The assessment of bone quality by quantitative ultrasound (QUS), a transportable and relatively cheap method, shows some correlations with bone mineral density (BMD) as measured by dual-energy X-ray absorptiometry (DXA) and with fracture risk. To examine its correlation with bone metabolism in a population of institutionalized elderly people known to be at high risk for vitamin D deficiency and secondary hyperparathyroidism, QUS of the calcaneus and biochemical parameters were measured in 264 women aged $85 \pm 7$ (SD) years and in 103 men aged $81 \pm 8$ years living in 19 nursing homes. Vitamin D deficiency was frequent in this population: $41.9 \%$ of the women and $31.4 \%$ of the men had a serum 25-hydroxyvitamin (25OHD) level below the 2.5th percentile level of 3276 normal Swiss adults $(6.2 \mu \mathrm{g} / \mathrm{l}$ or $15.5 \mathrm{mmol} / \mathrm{l})$. Hyperparathyroidism was less frequent: serum parathyroid hormone (PTH) levels were above the 97.5th percentile level of normal adults (70 pg/l) in 18.9\% of women and $9.8 \%$ of men. In women, QUS data correlated significantly with age $(r=-0.297)$, body mass index (BMI) $(r=0.403)$, calcium $(r=0.220)$, PTH $(r=-0.296), 250 H D(r=0.298)$ and alkaline phosphatase (AP) $(r=-0.170)$ for broadband ultrasound attenuation (BUA), and with age $(r=-0.195)$, BMI $(r=0.208)$, PTH $(r=-0.174), 25 \mathrm{OHD}(r=0.140)$ and AP ( $r=-0.130)$ for speed of sound (SOS). In men, ultrasound data correlated with BMI $(r=0.326)$, calcium $(r=0.199), 25 \mathrm{OHD}(r=0.258)$ and $\mathrm{AP}(r=-0.311)$ for
\end{abstract}

Correspondence and offprint requests to: $\mathrm{P}$. Burckhardt, MD, Department of Internal Medicine University Hospital - CHUV, CH1011 Lausanne, Switzerland. Tel: +41/21/31 40870. Fax: 41/21/31 40871 .
BUA, and with AP ( $r=-0.196)$ for SOS. In women, but not in men because of their smaller number, a multivariate analysis was performed to examine relationships between age, BMI, biochemical markers and QUS. Age, BMI, PTH and phosphate explained $30 \%$ of the variance of BUA and $10 \%$ for SOS. In conclusion, QUS of bone evaluates characteristics of bone that are influenced, at least partially, by age, BMI and the secondary hyperparathyroidism due to vitamin D deficiency.

Keywords: Institutionalized elderly; Secondary hyperparathyroidism; Ultrasound of bone; Vitamin D deficiency

\section{Introduction}

It has been shown that vitamin $\mathrm{D}$ deficiency is common in institutionalized elderly people [1-5]. This is explained by the decrease in endogenous production of vitamin $\mathrm{D}$ due to the lack of sun exposure, which is not balanced by an adequate vitamin D intake. The latter is lower than $200 \mathrm{IU} /$ day in the institutionalized elderly $[2,6]$ whereas the recommended intake is $400 \mathrm{IU} /$ day [7]. This situation leads to secondary hyperparathyroidism with an increased risk of fractures, principally hip fractures. Controlled studies showed a significant decrease in non-vertebral fractures after correction of the secondary hyperparathyroidism with supplements of vitamin $\mathrm{D}$ and calcium $[4,8]$.

Among the new techniques of bone assessment, quantitative ultrasound appears particularly promising. Recent studies showed that it was able to discriminate 
subjects with osteoporosis from those without osteoporosis [9-12]. In estimating the risk of hip fracture, it gave a similar positive predictive value to dual-energy X-ray absorptiometry (DXA) $[13,14]$.

Previous studies [15-17] showed that quantitative ultrasound of bone assessed parameters of bone quality other than density, such as three-dimensional bone structure for the broadband ultrasound attenuation (BUA) and bone elasticity for the speed of sound (SOS). But little is known about the relation between ultrasound data and the biochemical disturbances that are pathogenetic factors for osteoporosis, such as secondary hyperparathyroidism and vitamin $\mathrm{D}$ deficiency $[4,8,18]$. This study examined the potential relationships between the results of quantitative ultrasound of the calcaneus and biochemical markers of bone metabolism in a population of elderly people known to be at high risk for vitamin $\mathrm{D}$ deficiency and secondary hyperparathyroidism [1-5].

\section{Subjects and Methods}

Three hundred and forty-nine elderly persons living in 19 nursing homes around the University Hospital of Lausanne were studied. The exclusion criteria were specific treatments or diseases affecting bone metabolism, including calcium and vitamin D supplements, primary hyperparathyroidism and neoplasm with hypercalcemia, severe chronic diseases and poor vital prognosis (life expectancy less than 1 month). All subjects gave their written consent, and the study was approved by the ethics committee of the University of Lausanne.

All subjects were simultaneously assessed by quantitative ultrasound of their calcaneus and by a fasting serum analysis of bone metabolism parameters influenced by osteoporosis (albumin, calcium, phosphate, 25hydroxyvitamin D, parathyroid hormone (PTH), alkaline phosphatase and creatinine).

\section{Ultrasound of Bone}

Ultrasound measurements were performed at the calcaneus with an Achilles ultrasound apparatus (Lunar, Madison, WI). Attenuation (broadband ultrasound attenuation, BUA, expressed in $\mathrm{dB} / \mathrm{MHz}$ ) and speed (speed of sound, SOS, expressed in $\mathrm{m} / \mathrm{s}$ ) through the heel were measured. In a preliminary study [12], in which the reproducibility of the method was assessed, coefficients of variation were $1.8 \%$ for the BUA and $0.3 \%$ for the SOS. Controls with the manufacturer's phantom were performed throughout the study, as already reported [19]. They showed a good stability of the ultrasonic parameters.

\section{Biochemical Analysis}

Albumin, calcium, phosphate, creatinine and alkaline phosphatase concentrations were measured by standard laboratory methods. Serum 25-hydroxyvitamin D was measured by protein binding assay (Amsersham Life Science, Little Chalfont, Bucks, UK); the normal range for Swiss adults was: $6.2-42.0 \mu \mathrm{g} / \mathrm{l}$ (Swiss adults older than 65 years: $4.3-40.5 \mu \mathrm{g} / \mathrm{l}$ ) [20]. Serum intact parathyroid hormone (PTH) was measured by Allegro (Nichols Institute, San Juan Capristrano, CA); the normal range was $10-70 \mathrm{ng} / 1$ ).

\section{Statistical Analysis}

For comparison between independent groups, MannWhitney $U$-tests were performed. To assess the relationship between ultrasound and both anthropometric parameters and biochemical markers of bone metabolism a bivariate analysis (Spearman correlation coefficients) was performed first. Since these variables were likely to be correlated, and in order to find the amount of the variability of ultrasound explained by the parameters, we then performed a multiple regression analysis. Due to the relatively small number of men, this analysis was performed only in women. We included in the model all the variables from the bivariate analysis. The biochemical markers that were not normally distributed underwent natural logarithmic transformation to normalize the data. A stepwise procedure was used to identify all predictors that significantly added to the model's predictive power, using $p$ value of 0.05 for both entry and stay. The absence of collinearity between selected independent variables was assessed using the variance inflation factor. The model assumptions of normality and constant variance were tested by residual diagnostics.

\section{Results}

Anthropometric data of the population ( 246 women and 103 men), and the mean values of the quantitative ultrasound measurements of the calcaneus and of the biochemical markers, are shown in Table 1. The mean age was over 80 years in both sexes. Compared with the normal population, the mean values of 25 -hydroxyvitamin D were low in both sexes, while the mean PTH and alkaline phosphatase values were slightly above the normal mean. The plasma levels of 25 -hydroxyvitamin $\mathrm{D}$ were below the 2.5 th percentile $(6.2 \mu \mathrm{g} / \mathrm{l}$, i.e. 15.5 $\mathrm{nmol} / \mathrm{l}$ in $\mathrm{IU})$ of a large $(n=3276)$ Swiss adult general population [20] in $41.9 \%$ of the women and in $31.4 \%$ of the men. Compared with the general population aged $>65$ years, $28.8 \%$ of the women and $21.6 \%$ of the men were below the 2.5 th percentile $(4.3 \mu \mathrm{g} /$, i.e. 10.7 $\mathrm{mmol} / \mathrm{l}$ in IU). Concerning the serum level of $\mathrm{PTH}$, $18.9 \%$ of the women and $9.8 \%$ of the men were above the 97.5 th percentile $(70 \mathrm{pg} / \mathrm{l})$ of normal adults. 
Table 1. Anthropometric data, quantitative ultrasound of the calcaneus and biochemical markers



BUA, broadband ultrasound attenuation; SOS, speed of sound; PTH, parathyroid hormone.

${ }^{a}$ Corrected for albumin.

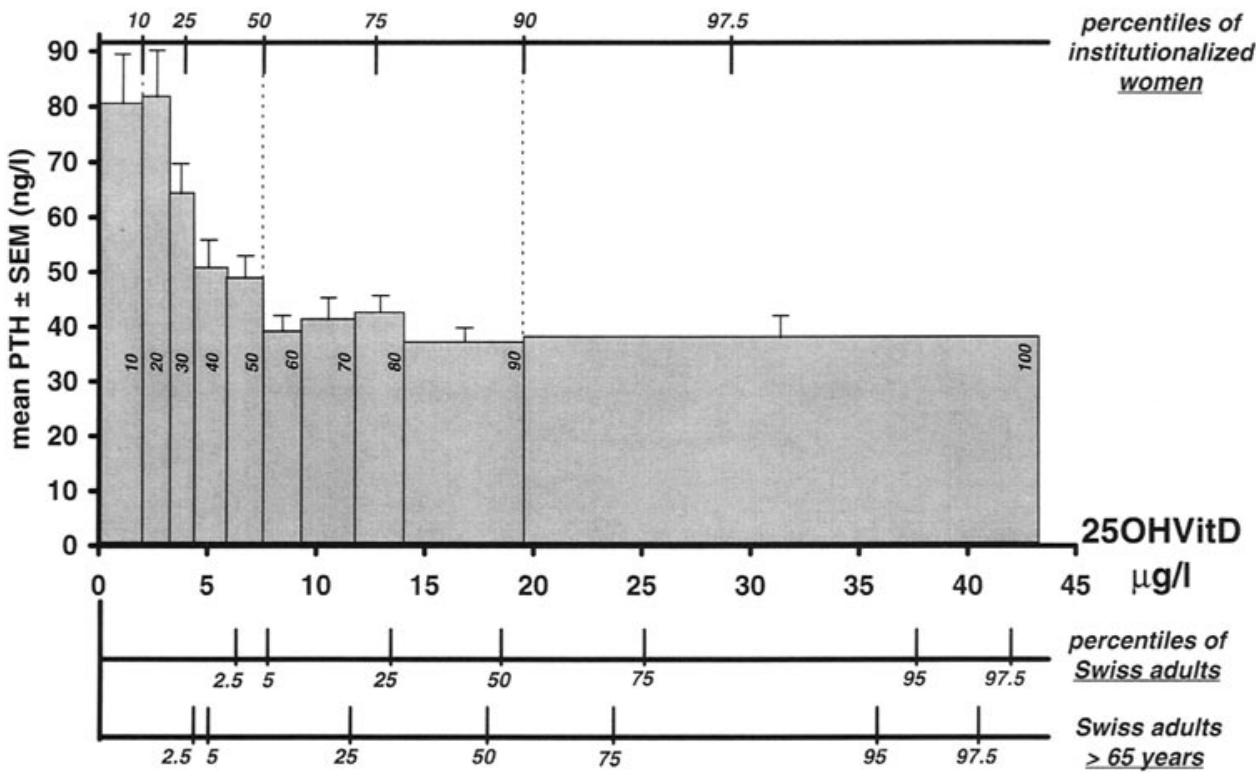

Fig. 1. Comparison of mean parathyroid hormone (PTH) values ( \pm SEM) and the 25-hydroxyvitamin D levels presented as groups of percentiles of a population of 246 institutionalized elderly women. For comparison, the percentiles of 25 -hydroxyvitamin D of the normal Swiss adult population (general population, $(n=3276$; people aged $>65$ years, $n=215$ ) are shown at the bottom. An increase in mean PTH is observed when 25 -hydroxyvitamin D levels are below $7.6 \mu \mathrm{g} / 1(19.0 \mathrm{mmol} / 1 \mathrm{in} \mathrm{IU})$, corresponding to the 50 th percentile for institutionalized elderly women but to the 5 th percentile for healthy Swiss adults.

After excluding subjects with serum creatinine higher than $120 \mu \mathrm{mol} / 1(n=46)$, the mean values of calcium and PTH levels remained unchanged; calcium $( \pm$ SD) values were $2.27 \mathrm{mmol} / \mathrm{l}( \pm 0.09)$ and $2.27 \mathrm{mmol} / \mathrm{l}( \pm$ $0.09)$, PTH $( \pm$ SD) values were $51.1 \mathrm{pg} / \mathrm{l}( \pm 31.3)$ and $49 \mathrm{pg} / \mathrm{l}( \pm 30.1)$ (NS) before and after this exclusion respectively.

Fig. 1 shows the mean PTH values as a function of the 25-hydroxyvitamin D levels for the women, presented by groups of percentiles. For comparison, the percentiles of 25-hydroxyvitamin D of the normal Swiss adult population, where no sex differences were found, are also shown [20]. In these elderly women, PTH increased progressively when the 25-hydroxyvitamin D levels were below $7.6 \mu \mathrm{g} / \mathrm{l}(19.0 \mathrm{nmol} / \mathrm{l}$ in IU), which corresponds to the 50th percentile of this group, and to the 5th percentile of the normal Swiss adult population. No such regular increase of PTH could be shown in men.

In women, PTH was negatively correlated with $25-$ hydroxyvitamin $\mathrm{D}(-0.485, p<0.001)$ and phosphate $(r=-0.188, p<0.01)$ and positively correlated with 
Table 2. Spearman rank correlation between quantitative ultrasound parameters and age, body mass index (BMI) and biochemical markers

\begin{tabular}{|c|c|c|c|c|c|c|c|}
\hline & Age & BMI & Calcium & Phosphate & PTH & $25 \mathrm{OHD}$ & $\mathrm{AP}$ \\
\hline \multicolumn{8}{|c|}{ Women } \\
\hline BUA & $-0.297^{* * * *}$ & $0.403^{* * * *}$ & $0.220 * * *$ & -0.087 & $-0.296 * * *$ & $0.298 * * *$ & $-0.170^{* * *}$ \\
\hline SOS & $-0.195^{* *}$ & $0.208 * * *$ & 0.057 & -0.051 & $-0.174 * *$ & $0.140^{*}$ & $-0.130^{*}$ \\
\hline \multicolumn{8}{|l|}{ Men } \\
\hline BUA & 0.117 & $0.326^{* * * *}$ & $0.199 *$ & 0.030 & -0.106 & $0.258^{* * *}$ & $-0.311^{* * *}$ \\
\hline SOS & 0.075 & 0.125 & 0.120 & 0.062 & -0.102 & 0.097 & $-0.196^{*}$ \\
\hline
\end{tabular}

PTH, parathyroid hormone; 25OHD, 25-hydroxyvitamin D; AP, alkaline phosphatase.

$* p<0.05, * * p<0.02, * * * * x<0.01$.

Table 3. Coefficients of the multiple regression models ( \pm SEE) explaining the ultrasound parameters in women $(n=246)$

\begin{tabular}{llllll}
\hline & Intercept & Age & BMI & PTH & Phosphate \\
\hline BUA & +134 & $-0.26 \pm 0.08$ & $+0.88 \pm 0.13$ & $-6.38 \pm 1.18$ & $-14.70 \pm 3.93$ \\
SOS & +1519 & $-0.62 \pm 0.25$ & $+1.12 \pm 0.38$ & $-7.86 \pm 3.29$ & NS \\
\hline
\end{tabular}

alkaline phosphatase $(r=0.206, p<0.01)$. 25-Hydroxyvitamin $\mathrm{D}$ was correlated with alkaline phosphatase $(r=0.285, p<0.0(0) 1)$. In men, PTH was negatively correlated with 25-hydroxyvitamin D $(-0.317$, $p<0.01)$, phosphate $(-0.265, p<0.01)$ and calcium $(r=-0.339 . p<0.001)$.

The correlations between age, body mass index, biochemical markers and the ultrasound measurements by bivariate analysis are presented in Table 2 . Significant predictors of BUA were age, body mass index, calcium, PTH. 25-hydroxyvitamin D and alkaline phosphatase in women, and body mass index, calcium, 25-hydroxyvitamin $\mathrm{D}$ and alkaline phosphatase in men. Age, body mass index, PTH, 25-hydroxyvitamin D and alkaline phosphatase were significant predictors of SOS in women, but with lower coefficients of correlation than for BUA, whereas in men, only alkaline phosphatase was a significant predictor of SOS.

In Table 3, the multiple regression analysis in women showed that age, body mass index, PTH and phosphate were independent significant predictors of BUA. Because of their correlation with PTH, 25-hydroxyvitamin $D$ and alkaline phosphatase were no longer independent predictors of BUA. According to $R^{2}, 30 \%$ of the variability of BUA was explained by this model. For SOS, only age, body mass index and PTH were independent significant predictors, with, according to $R^{2}$, only $10 \%$ of the variability in SOS being explained by this model.

\section{Discussion}

The aim of this study was to assess whether anthropometric parameters and biochemical markers of bone metabolism have a significant influence on quantitative ultrasound of bone in institutionalized elderly persons known to have a high percentage of vitamin $\mathrm{D}$ deficiency and secondary hyperparathyroidism. Indeed, the percen- tage of these metabolic disturbances was high, in agreement with the literature $[1-5,21]$, and was higher in women than in men. As expected. PTH was negatively correlated with 25-hydroxyvitamin D. In addition, in women, PTH and 25-hydroxyvitamin D were both correlated with alkaline phosphatase (the latter negatively), confirming the known interdependency of these markers. These correlations with alkaline phosphatase were not found by Brazier et al. [21] who assessed institutionalized elderly with a serum level of 25hydroxyvitamin $\mathrm{D}$ below $6 \mu \mathrm{g} / \mathrm{l}(15.6 \mathrm{nmol} / \mathrm{l})$. In a subgroup analysis in women with a serum level of 25 hydroxyvitamin $\mathrm{D}$ below $6 \mu \mathrm{g} / \mathrm{l}$, we still found significant correlations. This discrepancy might be explained by the smaller number of subjects examined by Brazier et al. The correlation between PTH and 25hydroxyvitamin D showed that the mean PTH level increased when the 25-hydroxyvitamin $\mathrm{D}$ level dropped below $7.6 \mu \mathrm{g} / 1(19.0 \mathrm{nmol} / \mathrm{l})$, and rose above $70 \mathrm{ng} / \mathrm{l}$ (the upper normal value of our laboratory) at a 25 hydroxyvitamin D level of $3.4 \mu \mathrm{g} / \mathrm{l}(8.9 \mathrm{nmol} / \mathrm{l})$. This is in partial agreement with the observations in an adult normal population by Chapuy et al. [22] who showed that PTH rose above $55 \mathrm{ng} / \mathrm{l}$ (their upper normal limit) when the 25-hydroxyvitamin D level was below $4.6 \mu \mathrm{g} / \mathrm{l}$ (11.3 nmol/l) [22].

The ultrasound measurements were low when compared with other studies, mainly due to the advanced age of the subjects [19] (mean $T$-score for women as defined by the manufacturer: $\mathrm{BUA}=57 \%, \mathrm{SOS}=47 \%$; mean $T$-score for men: $\mathrm{BUA}=75 \%, \mathrm{SOS}=61 \%$ ). They were also found to be linked to the disturbances of bone metabolism related to older age: the bivariate analysis revealed that in addition to the already known negative influence of age and positive influence of BMI $[19,23-$ 25] all major markers were correlated with BUA, i.e. 25-hydroxyvitamin $\mathrm{D}$, alkaline phosphatase and to a lesser degree calcium, in both sexes, and PTH in women. 
This points to a rather non-specific reflection of bone mass and structure by BUA, as influenced by the various known pathogenic factors of osteoporosis in advanced age. The absence of correlation with PTH in men is probably explained by the smaller number of subjects. It might also be explained by the lower incidence of secondary hyperparathyroidism in men even though there is no reason to assume that bone metabolism in advanced age is sex dependent. In essence, men showed the same trend in correlation between markers and BUA, probably less significantly because of the smaller number.

SOS correlated in the women with all parameters in the same order as the correlations with BUA, but less significantly. In men all the correlations of BUA were less significant than in women. SOS was not significantly correlated with any of the biological parameters, except alkaline phosphatase. SOS is supposed to measure a quality of bone other than that measured by BUA, but its relation to the biochemical profile is the same as that of BUA, only less evidently.

The correlations are comparable to those found between biochemical markers and bone mineral density of the femoral neck as measured by DXA. Although Chapuy et al. [26] did not find any correlation between 25-hydroxyvitamin D or PTH and hip BMD, Ooms et al. [27] found a correlation coefficient between BMD of the femoral neck and PTH of $-0.270(p<0.001)$ in 330 women aged \pm 80.3 years. Martinez et al. [28] found a correlation between 25-hydroxyvitamin D and BMD of the femoral neck with a correlation coefficient of 0.290 $(p<0.005)$ in 62 women aged more than 68 years. Szluc et al. [29] published data on the relationship between hip BMD and anthropometric parameters and biochemical markers in 98 elderly institutionalized women aged \pm 81.4 years. The correlation coefficients were -0.41 to -0.47 for age $(p<0.001), 0.39$ to 0.51 for body weight $(p<0.001),-0.27$ to -0.33 for PTH $(p<0.001)$, -0.21 to -0.33 for alkaline phosphatase $(p<0.005)$ and -0.01 to -0.09 (NS) for 25 -hydroxyvitamin D. This leads to the assumption that DXA and quantitative ultrasound reflect partially the same pathogenetic factors, although probably measuring different characteristics of bone. Age and body weight showed the highest correlations, followed by PTH. The absence of a significant correlation with 25 -hydroxyvitamin $\mathrm{D}$ in this study can be explained by the relatively small number of subjects examined. From these studies it can be concluded that there is no essential difference between BUA of the heel and BMD of the hip as measured by DXA regarding their correlations with anthromometric and biochemical parameters.

In women, a multivariate regression analysis was performed to examine more specific relationships between biochemical markers and quantitative ultrasound. This analysis explained $30 \%$ of the variance of BUA and $10 \%$ of the variance of SOS by the evaluated parameters.

The multivariate regression analysis showed that secondary hyperparathyroidism expressed by PTH level was an independent predictor of BUA. However, neither 25-hydroxyvitamin D nor alkaline phosphatase was an independent predictor. This allows the conclusion that bone quality evaluated by BUA reflects the effect of the elevated PTH level rather than that of the lower 25-hydroxyvitamin D. Surprisingly, phosphate was an independent negative predictor of BUA, although it showed no significant correlation with any of the markers (except with PTH) or with BUA in the bivariate analysis. Phosphate levels were normal or low in this population, and a positive correlation with BUA could be expected since low phosphate levels reveal malnutrition and/or vitamin D deficiency, both potentially contributing to a low BUA. It can only be hypothesized that a relatively high serum phosphate level (there was no hyperphosphatemia) revealed a more advanced reduction in renal function and by this a tendency to secondary hyperparathyroidism, but no such correlations could be seen in the bivariate analysis.

In conclusion, the quantitative ultrasound parameter BUA, and to a much smaller degree SOS, evaluates the characteristics of bone that are influenced mainly by age, BMI and secondary hyperparathyroidism. In order to support these observations, further investigations are needed to examine the question of whether the reduction of secondary hyperparathyroidism leads to an improvement in BUA.

Part of this work was presented at the 17 th Annual Meeting of the American Society of Bone and Mineral Research in Baltimore, MA, USA, September 1995.

\section{References}

1. Corless D, Gupta SP, Sattar DA, Switala S, Boucher BJ. Vitamin D status of residents of an old people's home and long-stay patients. Gerontology 1979;25:350-5.

2. Chapuy MC, Chapuy P, Meunier PJ, Calcium and vitamin D supplements: effects on calcium metabolism in elderly people. Am J Clin Nutr 1987;46:324-8.

3. Lips P, Wiersinga A, Van Ginkel FC, Jongen MJM, Netelenbos JC, Hackeng WHL, et al. The effect of vitamin D supplementation on vitamin $D$ status and parathyroid function in elderly subjects. J Clin Endocrinol Metab 1988;67:644-50.

4. Heikinheimo RJ, Inkovaara JA, Harju EJ, Haavisto MV, Kaarela RH, Kataja JM, et al. Annual injection of vitamin D and fractures of aged bones. Calcif Tissue Int 1992;51:105-10.

5. Deroisy $R$, Zheng XS, Pirenne $H$, Frederick I, Collette $J$, Reginster JY. 25-Hydroxyvitamin D deficiencies and secondary hyperparathyroidism in elderly women living at home or in nursing/retirement homes. Osteoporos Int 1996;6(Suppl 1):119.

6. Burckhardt P, Gerber S, Jacquet AF, Thiébaud D. Lack of fish is the main cause of vitamin D deficiency in institutionalized elderly swiss subjects. J Bone Miner Res 1993;8(Suppl 1):S218.

7. Recommended Dietary Allowances. 9th ed. Food and Nutrition Board, Nutritional Research Council. Washington, DC: National Academy of Sciences, 1980.

8. Chapuy MC, Arlot ME, Duboeuf F, Brun J, Crouzet B, Arnaud S, et al. Vitamin $D_{3}$ and calcium to prevent hip fractures in elderly women. N Engl J Med 1992;327:1637-42.

9. Lees B, Stevenson JC. Preliminary evaluation of a new ultrasound bone densitometer. Calcif Tissue Int 1993;53:149-52.

10. Yamasaki K, Kushida K, Ohmura A, Sano M, Inoue $T$. Ultrasound bone densitometry of the os calcis in Japanese women. Osteoporos Int 1994;4:220-5. 
11. Schott AM, Weill-Engerer S, Hans D, Duboeuf F, Delmas PD, Meunier PJ. Ultrasound discriminates patients with hip fracture equally well as dual energy X-ray absorptiometry and independently of bone mineral density. J Bone Miner Res 1995;10:243-9.

12. Krieg MA, Thiébaud D, Landry M, Burckhardt P. Evaluation de la qualité osseuse par ultrason quantitatif. Schweiz Med Wochenschr 1996;126:159-63.

13. Bauer DC,Glüer CC, Pressman AR, Vogt TM, Ensrud KE, Cauley JA, et al. Broadband ultrasonic attenuation (BUA) and the risk of fracture: a prospective study. J Bone Miner Res 1995;10(Suppl 1):S175.

14. Hans D, Dargent-Molina P, Schott AM, Sebert JL, Cormier C, Kotzki PO, et al. Ultrasonographic heel measurements to predict hip fracture in elderly women: the EPIDOS prospective study. Lancet 1996;348:511-4.

15. Ashman RB, Cowin SC, Van Buskirk WC, Rice JC. A continuous wave techique for measurement of elastic properties of cortical bone. J Biomech 1984;17:349-61.

16. Glüere $\mathrm{CC}, \mathrm{Wu} \mathrm{Cy}$, Genant $\mathrm{HK}$. Broadband ultrasound attenuation signals depend on trabecular orientation: an in vitrol study. Osteoporos Int 1993;3:185-91.

17. Hins D, Arlot ME, Schott AM, Roux JP, Kotzki PO, Meunier PJ. Do ultrasound measurements on the os calcis reflect more the bone microarchitecture than the bone mass? A two-dimensional histomorphometric study. Bone 1995;16:295-300.

18. Watern E, Christiansen C, Gause-Nilsson I, G. Lindstedt, Oden A, Riis B, Mellström D. Calcidiol (25-OH vitamin D) is a predictor of bone mineral density and hip fracture. Osteoporos Int 1996:6(Suppl 1):127.

19. Krièg MA, Thiébaud D, Burckhardt P. Quantitative ultrasound of bonc in institutionalized elderly women: a cross-sectional and longitudinal study. Osteoporos Int 1996;6:189-95.

20. Burnand B, Sloutskis D, Gianoli F, Cornuz J, Riggenbach $M$, Paccatd F, Burckhardt P. Serum 25- $\mathrm{OH}$ vitamin D: distribution and determinants in the Swiss population. Am J Clin Nutr $1992 ; 56: 537-42$.
21. Brazier M, Kamel S, Maamer M, Agbomson F, Elesper I, Garabedian M, et al. Markers of bone remodeling the elderly subject: effects of vitamin D insufficiency and its correction. J Bone Miner Res 1995;10:1753-61.

22. Chapuy MC, Preziosi P, Maamer M, Arnaud S, Galan P, Hercberg S, Meunier PJ. Prevalence of vitamin D insufficiency in an adult normal population. Osteoporos Int 1997;7: in press.

23. Schott AM, Hans D, Sornay-Rendu E, Delmas PD, Meunier PJ. Ultrasound measurements on os calcis: precision and age-related changes in a normal female population. Osteoporos Int 1993;3:249-54.

24. Van Daele PLA, Burger H, Algra D, Hofman A, Grobbe DE, Birkenhäger JC, Pols HAP. Age-associated changes in ultrasound measurements of the calcaneus in men and women: the Rotterdam study. J Bone Miner Res 1994;9:1751-7.

25. Yamasaki K, Kushida K, Ohmura A, Sano M, Inoue $T$. Ultrasound bone densitometry of the os calcis in Japanese women. Osteoporos Int 1994;4:220-5.

26. Chapuy MC, Schott AM, Garnero P, Hans D, Delmas PD, Meunier PJ, and the EPIDOS Study Group. Healthy elderly French women living at home have secondary hyperparathyroidism and high bone turnover in winter. J Clin Endocrinol Metab 1996;81:1129-33.

27. Ooms ME, Lip P, Roos JC, van der Vijgh WJ, Popp-Snijders C, Bezemer PD, Bouter LM. Vitamin D status and sex hormone binding globulin: determinants of bone lurnover and bone mineral density in elderly women. I Bone Miner Res 1995;10:1177-84.

28. Martine\% ME, del Campo MT, Sanches-Caberudo MJ, Garcia JA, Sanchez-Calvin MT, Torrijos A, et al. Relations between calcidiol, serum levels and bone mineral density in postmenopausal women with low bone density. Calcif Tissue lnt 1994;55:253-6.

29. Srluc P, Arlot M, Chapuy MC, Dubout F, Meunier PJ, Delmas PD. Serum undercarboxylated osteocalcin correlates with hip bone mineral density in elderly women. J Bone Miner Res $1994 ; 9: 1591-5$. 